\title{
Interculturalidad en lenguas-culturas extranjeras: un desafío filosófico para América Latina ${ }^{1}$
}

\author{
Interculturality in foreign languages-cultures: \\ a philosophical challenge for Latin America
}

Jorge Hernán Herrera Pineda ${ }^{2}$

Claudia Elizabeth Ortiz Ruiz ${ }^{3}$

Universidad del Quindío, Colombia

Recepción: 10 de enero del 2018

Evaluación: 13 de marzo del 2018

Aceptación: 25 de junio del 2018

1 El presente artículo es el resultado del proyecto, con código 821, titulado "Colonialidad del saber y del ser en las concepciones de interculturalidad del Programa de Licenciatura en Lenguas Modernas de la Universidad del Quindío. Proyecto adelantado por el grupo de investigación Esapidex-B del Programa de Licenciatura en Lenguas Modernas de la Universidad del Quindío.

2 Jorge Hernán Herrera es licenciado en lenguas modernas de la Universidad del Quindío, magíster en didáctica del francés como lengua extranjera de la Universidad del Rosario, especialista en filosofía de la ciencia de la Universidad El Bosque y especialista en alta gerencia de la Universidad Industrial de Santander. Además, ha publicado el artículo de investigación científica "Aproximación filosófica a la didáctica de las lenguas-culturas: la fenomenología en la perspectiva accional", Revista Lenguaje, 2016, 44 (2).

Correo electrónico: jhherrera@uniquindio.edu.co.

3 Claudia Elizabeth Ortiz es licenciada en lenguas modernas de la Universidad del Quindío, magíster en didáctica del francés, Université Jean Monnet, Francia..

Correo electrónico: ceortiz@uniquindio.edu.co.

Herrera, J \& Ortiz, C. (2018). Interculturalidad en lenguas-culturas extranjeras: un desafío

filosófico para América Latina. Cuestiones de Filosofía, 4 (22), 173-199.

doi: https://doi.org/10.19053/01235095.v4.n22.2018.8301 


\section{Resumen}

Este artículo de reflexión problematiza la didáctica de las lenguas extranjeras desde una inquietud filosófica-intercultural para contextos mayoritaria e históricamente monolingües como el latinoamericano. Inicialmente, se describen los rasgos de colonialidad latentes en el enfoque que el Marco Común Europeo de Referencia asume para tratar la interculturalidad. Seguidamente, se realiza un análisis crítico-filosófico de los desafíos que la interculturalidad enfrenta en América Latina en el contexto anteriormente mencionado, y se revisan las relaciones que existen entre interculturalidad y glotopolítica, asumiendo que el latinoamericanismo de Mendieta, la resemantización del orientalismo de Said, el mimetismo y la hibridación bhabhiana, así como la filosofía intercultural, son quizá los desafíos filosóficos más grandes. Finalmente, se presenta un exordio para una interculturalidad crítica en lenguas-culturas extranjeras, en el que se tienen en cuenta algunas contribuciones del Grupo Modernidad/Colonialidad, concretamente de Dussel, Walsh, Castro-Gómez y Fornet-Betancourt, reconociendo que la didáctización de la interculturalidad en las licenciaturas de lenguas modernas de Colombia está por construirse.

Palabras clave: colonialidad, didáctica, lengua extranjera, cultura, interculturalidad, filosofía.

\section{Abstract}

This paper of reflection problematizes the didactic of foreign languages from a philosophical-intercultural framework worry in contexts that are mostly and historically monolinguals, as it happens in the Latin-American one. Initially, it is described the features of colonialism that are present in the approach that the Common European Framework of Reference assumes to deal with interculturality. Afterward, it is performed a critic-philosophic analysis of the challenges that the interculturality deals in Latin America in the context previously mentioned. Reviewing the relationships that exist between interculturality and glotopolitics, assuming that the LatinAmericanism of Mendieta, the re-semanticization from the orientalism of Said, the bhabhian mimesis and hybridization and, likewise, the intercultural philosophy are perhaps the greatest philosophical challenges. Finally, it's presented a preamble for a critic interculturality in foreign languages-cultures, considering some of the contributions of the Modernity/Coloniality group, 
particularly from Dussel, Walsh, Castro-Gomez, and Fornet-Betancourt. Acknowledging that the "didactication" of the interculturality is still to be built in the bachelor degrees of modern languages of Colombia.

Keywords: coloniality, didactic, foreign language, culture, interculturality, philosophy.

\section{Interculturalité en langues-cultures étrangères: un défi philosophique pour l'Amérique Latine.}

\section{Résumé}

Cet article de réflexion pose la problématique de la didactique des langues étrangères dans des contextes majoritairement et historiquement monolingues tel que celui de l'Amérique Latine, du point de vue d'une inquiétude philosophique-interculturelle. Pour commencer, les traits de la colonialité, latents dans l'approche que le Cadre Européen Commun de Référence prend pour aborder l'interculturalité, sont décrits. Ensuite, une analyse critiquephilosophique des défis auxquels l'interculturalité fait face en Amérique Latine dans le contexte susmentionné est faite, basée sur une révision des relations existantes entre interculturalité et glottopolitique, partant du principe que le "latinoamericanismo de Mendieta", la resemantisation de l'orientalisme de Said, le mimétisme et l'hybridation bahbhienne, ainsi que la philosophie interculturelle, sont probablement les plus grands défis philosophiques. Enfin, un exodium pour une interculturalité critique en langues-cultures étrangères est présenté, tenant compte de certaines contributions du Groupe Modernité/ Colonialité, dont concrètement Dussel, Walsh, Castro-Gómez et FornetBetancourt, reconnaissant que la didactisation de l'interculturalité dans les licences en langues modernes en Colombie reste à construire.

Mots-clés: colonialité, didactique, langue étrangère, culture, interculturalité, philosophie. 


\section{Interculturalidade nas línguas-culturas estrangeiras: um desafio filosófico para América Latina}

\section{Resumo}

Este artigo de reflexão problematiza a didática das línguas estrangeiras desde uma inquietude filosófica-intercultural para contextos historicamente monolíngues como o latino-americano. Inicialmente, se descrevem os rasgos de colonialidade latentes no enfoque que o Marco Comum Europeio de Referencia assume para tratar a interculturalidade. Seguidamente, se realiza uma análise critico-filosófico dos desafios que a interculturalidade enfrenta em América Latina no contexto anteriormente mencionado, revisando as relações que existem entre interculturalidade e glotopolítica, assumindo que o latinoamericanismo de Mendieta, a resemantização do orientalismo de Said, o mimetismo e a hibridação bhabhiana, assim como a filosofia intercultural são talvez os maiores desafios filosóficos. Finalmente, se apresenta um exordio para uma interculturalidade crítica em línguas-culturas estrangeiras, tomando em conta algumas contribuições do Grupo Modernidade/Colonialidade, concretamente de Dussel, Walsh, Castro-Gómez e Fornet-Betancourt, reconhecendo que a didactização da interculturalidade nas licenciaturas de línguas modernas de Colômbia está por se construir.

Palavras-chave: colonialidade, didática, língua estrangeira, cultura, interculturalidade, filosofia. 


\section{Introducción}

Este trabajo pretende establecer los desafíos filosóficos a los que se ve expuesta la didáctica de la interculturalidad en la enseñanza-aprendizaje de las lenguas-culturas extranjeras en contextos lingüística, histórica y políticamente complejos como el de América Latina ${ }^{4}$. Al referirnos a lenguas-culturas extranjeras, estamos tomando en cuenta lo que Mignolo (2013) define como lenguas hegemónicas e imperiales, que Chardenet (2016) representa como internacionales: el inglés, el francés, el alemán, el portugués y el español mismo. Establecer criterios didácticos sobre interculturalidad desde una perspectiva europea o eurocéntrica, es, según nuestro criterio, un desacierto educativo y, consecuentemente, se convierte en un problema epistemológico que para resolverse debe examinarse no solo desde una perspectiva didáctica, sino también desde una inquietud filosófica; sobre todo, si esta pretende una autodeterminación en la didáctica de la interculturalidad en lenguas-culturas extranjeras en América Latina.

Con el propósito de constituir una autonomía intercultural estableciendo distancias críticas en relación con la epistemología eurocéntrica propia a la colonialidad del saber que acusa el Grupo Modernidad/Colonialidad, este trabajo de reflexión se soporta en una interpretación filosófica de autores que han participado en el grupo previamente referido y en algunas reflexiones provenientes de la teoría postcolonial, con el fin de exponer una ruta intercultural construida en tres fases.

Inicialmente, se analizan las expresiones de colonialidad del saber intercultural que se desprenden del Marco Común Europeo de Referencia (en adelante MCER, 2002) y que, desde nuestra perspectiva, son parcialmente pertinentes para contextos mayoritariamente monolingües por cuenta de procesos glotofágicos (Calvet, 1999, citado en Montoya, 2013) como es el caso de América Latina.

En segundo lugar, se hace un análisis crítico-filosófico de los desafíos que enfrenta la interculturalidad en América Latina, contexto en el cual han incidido y siguen incidiendo elementos conexos a la colonialidad del poder, del

\footnotetext{
4 Este trabajo de reflexión proviene de la fase de revisión de la literatura del proyecto de investigación "Colonialidad del saber y del ser en las concepciones de interculturalidad en el currículo oficial del Programa de Licenciatura en Lenguas Modernas de la Universidad del Quindío", financiado por la Universidad del Quindío y adscrito al grupo de investigación ESAPIDEX-B.
} 
saber y del ser, que dan como resultado la representación errónea del mestizaje para resolver la diversidad identitaria del continente (Fornet-Betancourt, 2004) al obviar a sus pueblos indígenas y afroamericanos.

En tercer lugar, se presenta un exordio de interculturalidad crítica que pretende construir una ruta mediante la cual la didáctica de la interculturalidad en la enseñanza-aprendizaje de lenguas-culturas extranjeras comience a expresarse con espíritu reivindicativo, crítico-constructivo y, a la vez, dialógico con quienes de buena fe y desde Europa han teorizado sobre la didáctica intercultural, pero que desconocen en su afán educativo las complejas realidades lingüísticas, históricas y políticas de los mundos que existen y cohabitan en América Latina.

A continuación, se problematiza la didáctica de las lenguas extranjeras desde una inquietud filosófica-intercultural para contextos latinoamericanos.

\section{La didáctica de la interculturalidad en lenguas extranjeras según el Marco Común Europeo de Referencia}

Para abordar la interculturalidad desde una inquietud didáctica-contemporánea en lenguas extranjeras, se tomaron en cuenta las competencias que se describen en el MCER (2002) y el "Cadre de Référence pour les Aproches Plurielles des Langues et des Cultures", (Marco de Referencia de Enfoques Pluralistas de las Lenguas y las Culturas, en adelante CARAP) (Candelier, 2012), ambos documentos creados por el Consejo de Europa para su respectiva población.

El CARAP tiene como propósito proveer a los maestros la facilidad de acceder a materiales que les permitan implementar actividades en el aula, para ayudar a los estudiantes a apropiarse de las habilidades y conocimientos mediante enfoques pluralistas en el proceso de enseñanza-aprendizaje de diversas lenguas.

Por su parte, el MCER tiene dos objetivos. El primero apunta a fomentar en todas las personas que estén en contacto con las lenguas, desde una perspectiva profesional o educativa, una serie de reflexiones acerca de las capacidades para actuar de manera eficaz en presencia del otro diverso y hacer el papel de usuarios de una lengua, la evaluación de los progresos en las lenguas extranjeras o segundas, el aprendizaje de las lenguas y su perfeccionamiento. El segundo objetivo del MCER consiste en facilitar -a aquellos usuarios de las 
lenguas que hacen parte de un mercado laboral europeo y que deben viajar o instalarse en países en donde las lenguas que se hablan no corresponden con las suyas- a comunicarse entre sí y, por consiguiente, a gestionar de manera eficaz las necesidades de sus clientes, objetivo intercultural del MCER.

El MCER afirma que la enseñanza-aprendizaje de las lenguas extranjeras debe abordarse desde lo que se reconoce como competencias comunicativas de la lengua, es decir, las competencias lingüísticas, las sociolingüísticas y las pragmáticas. Taxonómicamente, la interculturalidad se ubica al interior de las competencias sociolingüísticas que el MCER define como "las condiciones socioculturales del uso de la lengua. Mediante la sensibilidad a las convenciones sociales (...), el componente sociolingüístico afecta considerablemente a toda la comunicación lingüística entre representantes de distintas culturas, aunque puede que los integrantes a menudo no sean conscientes de su influencia" (2002, p.13).

La competencia sociolingüística implica a su vez el reconocimiento de una competencia plurilingüe y pluricultural, lo que se comprende como "la capacidad de utilizar las lenguas para fines comunicativos y de participar en una relación intercultural en que una persona, en cuanto agente social, domina (...) varias lenguas y posee experiencias de varias culturas" (p.166). Bajo este presupuesto, el MCER (2002) considera que un individuo con un alto desarrollo de competencia plurilingüe y pluricultural es capaz de tomar distancias del equilibrio que establece el bilingüismo entre una L1 (lengua materna) y una L2 (lengua extranjera). En su lugar, establece un interés por el plurilingüismo, lo cual no quiere decir que, porque dicho sujeto logra desarrollar un buen dominio lingüístico comunicativo, logra desarrollar igualmente la capacidad para relacionarse con otras culturas.

En ese sentido, al hablarse de interculturalidad en un ámbito didáctico y sociolingüístico, se deduce que el sujeto hablante de una lengua extranjera se convierte en plurilingüe y, por consiguiente, "desarrolla una interculturalidad" (MCER, 2002, p.46). En otras palabras -al concebir al usuario de la lengua, que para los efectos de la enseñanza-aprendizaje de las lenguas extranjeras en contextos mayoritariamente monolingües son estudiantes- se generan entonces una serie de destrezas, capacidades y conciencia intercultural en presencia de las competencias lingüísticas y culturales a las que se ve abocado al aprender otra(s) lengua(s). 
El MCER define la consciencia intercultural (a veces con "s" intermedia, otras veces no) como "el conocimiento, la percepción y la comprensión de la relación entre 'el mundo de origen' y el 'mundo de la comunidad objeto de estudio' (...) [que] producen una consciencia intercultural” (2002, p.101). Esa consciencia implica diversidad que tiende a enriquecerse por cuenta del encuentro de la lengua materna con otras lenguas; igualmente, se produce una toma de consciencia de los clichés que suelen categorizar a unos y otros según su procedencia.

La consciencia intercultural, en calidad de competencia comunicativa, está construida a partir de una taxonomía de habilidades y destrezas que se pueden resumir en capacidades para establecer relaciones entre la cultura de origen y la extranjera, la sensibilidad cultural que implica estrategias para ponerse en contacto con otros que provienen de otras culturas, la capacidad para hacer las veces de intermediario cultural y la capacidad para superar estereotipos.

Según el MCER (2002), comprometerse con la competencia intercultural implica a su vez reconocer igualmente que se puede llegar a desarrollar una personalidad intercultural, lo que supera la dimensión de las competencias y se adentra en las categorías educativas. En ese contexto de discusión, se hace necesario preguntarse por cuestiones como la ética, la moral y el relativismo cultural.

Por otro lado, el MCER establece diferencias entre conocimiento sociocultural y desarrollo de destrezas interculturales. En ese propósito, recurre a la similitud que algunos pueblos europeos comparten, pero, a la vez, a la diversidad entre naciones, regiones, comunidades étnicas representadas en estereotipos que poco favorecen la realidad de la vida de los habitantes de diferentes contextos culturales. En tal sentido, el MCER recomienda que -en los propósitos educativos arriba mencionados (ética intercultural, moral y relativismo cultural)- es importante encontrar un equilibrio que conduzca al estudiante a superar esos estereotipos. En todo caso, al reflexionar a propósito de la diversidad, la similitud y los estereotipos de las diferentes culturas, el documento referido sólo toma como punto de discusión geográfico a Europa.

Más allá de los aportes didácticos y epistemológicos del MCER, en lo que concierne a la interculturalidad, hay otros autores que han estudiado desde Europa a profundidad el término y lo han problematizado desde diferentes intereses. Lázár et al. (2007) plantean, a partir de lo que ellos definen como competencia comunicativa intercultural, tres dimensiones: 
- El saber que se comprende como los conocimientos que adquiere el estudiante y que le permiten reconocer la memoria colectiva en términos de cultura y civilización (enfoque humanístico), los conocimientos relacionados con la diversidad de modos de vida de las diferentes culturas (enfoque antropológico) y los elementos relacionados con los contextos socioculturales de las sociedades extranjeras (enfoque sociológico).

- El saber-hacer que da cuenta de la competencia lingüística y de comunicación del estudiante que le permite funcionar e interactuar en la lengua extranjera, la manera como aquél se ajusta a los ambientes sociales y culturales para adaptarse, integrar, negociar e interpretar en diversos contextos interculturales.

- El saber-ser que se orienta a la toma de conciencia crítico-cultural del estudiante, cuyo interés se concentra en la comprensión de las diferencias y similitudes entre culturas, al considerar, de esta manera, otras identidades, otras creencias y valores a partir de los propios, para así generar en el estudiante la idea de un intermediario intercultural.

El MCER está construido a partir de una epistemología basada no sólo en un pensamiento de la representación -que, según Deleuze (1985, citado en Martínez, 2009), se comprende como un pensamiento objetivo centrado en el aprendizaje concebido como instrumento único- sino también en un pensamiento abismal. Este último, definido por Santos (2010) como aquella epistemología visible y útil en contraste con su exacto contrario, expresado en la inexistencia y en la subsiguiente imposibilidad de comprensión o de coexistencia de otras formas de comprensión e interpretación del mundo, de la realidad y, por consiguiente, de la interculturalidad.

El pensamiento abismal es quizá la más latente forma de exclusión ontológica de carácter epistemológico. Santos la concentra en la modernidad occidental, cuyas consecuencias se manifiestan en regulación social concebida para el lado de la línea visible y en emancipación social para el otro lado de la línea no visible, lo cual impacta de manera directa a los territorios coloniales. En su conformación sólo existe Europa y aunque en ningún momento el MCER se expresa doctrinariamente como panacea didáctica para el resto del mundo, sus creadores y los respectivos usuarios saben que se globalizó.

Cuando el docente de lenguas-culturas extranjeras, en contexto mayoritariamente monolingüe, didactiza la interculturalidad, normalmente recurre al 
MCER y a autores europeos o anglosajones que, desde sus propias vivencias y perspectivas, proponen interpretaciones que se asumen como acertadas y se articulan en el logro de competencias de la lengua. Esa adopción epistemológica y didáctica - proveniente de Europa como centro de pensamiento y racionalidad en el campo de las lenguas extranjeras- es una admisión eurocéntrica de la interculturalidad, un consentimiento con un pensamiento abismal. Si la didáctica de las lenguas extranjeras que se enseñan en América Latina no estructura una idea de interculturalidad desde una visión crítica, histórica, social y política, tanto global como local, se corre el riesgo de acogerse a nuevas formas de colonialidad epistemológica y ontológica que deben problematizarse para la didáctica de la interculturalidad en lenguas extranjeras en contexto mayoritariamente monolingüe.

\section{Análisis crítico-filosófico de los desafíos que enfrenta la interculturalidad en América Latina}

Al reflexionar sobre interculturalidad para un continente como el de América Latina, la primera inquietud que surge es definir si ésta es una competencia, un concepto, una idea, una postura o disposición (Fornet-Betancourt, 2004) política y/o histórica, una forma de acceso a la interpretación de las referencias identitarias o algo completamente nuevo.

Como ya establecimos en el punto precedente, para el MCER, la interculturalidad es una competencia comunicativa que logra desarrollar las competencias lingüísticas, sociolingüísticas y pragmáticas. Este documento, base para la enseñanza-aprendizaje de las lenguas extranjeras a nivel mundial, es una evidente expresión de colonialidad del saber. La interculturalidad para la didáctica de las lenguas extranjeras existe en el seno del pensamiento europeo, está inmersa y hace parte de la estructura formativa que debe procurarse en los estudiantes de dichas lenguas, a quienes se les advierte como usuarios o actores sociales que establecen contacto con la otredad en el contexto real sociolingüístico de esas lenguas.

Un escenario didáctico resulta problemático para la didáctica de las lenguas-culturas extranjeras en contextos que por incidencias glotofágicas se han convertido desde 1492 en mayoritariamente monolingües. Calvet dice que en este contexto "solo hay una lengua oficialmente reconocida, la cual es el instrumento de comunicación mayoritario del Estado, la escuela, los medios de comunicación, etc" (1999, citado en Montoya, 2013, p.19). Por su 
parte, Flores Farfán (2010, citado en Plaza, 2016) afirma que, en los contextos en donde se han producido procesos de independencia y posterior revolución, se han evidenciado efectos glotofágicos que siguen manifestándose en el presente, lo que genera como consecuencia la depreciación de lenguas y culturas indígenas hasta gestarse una ideología monolingüe y monocultural.

Consintiendo con el fenómeno de la glotofágia, hay que admitir igualmente, como lo afirma Plaza (2016), que en estos contextos se presenta el fenómeno del desplazamiento lingüístico, que se caracteriza por una dinámica en la cual hay progresión y regresión de lenguas a partir de las relaciones de poder que allí se establecen. De acuerdo con el autor, la lengua en progresión se instala al interior de la comunidad dominante, lo que históricamente suele concluir en un contexto mayoritariamente monolingüe, mientras que la lengua en regresión es hablada por la comunidad subordinada y lingüísticamente frágil.

Mignolo (2013) advierte que las lenguas que se instalaron y posteriormente substituyeron aquellas manifestaciones plurilingües que existían hasta el momento en que aparecieron las campañas de conquista europeas, generaron ideologías propias a estructuras monolingües, como lo declara Flores Farfán, ajustadas a una hermenéutica monotópica que Mignolo (2009) comprende como aquella que unívocamente habla en nombre del resto de la humanidad. De igual forma, en el contexto mayoritariamente monolingüe, las lenguas ancestrales y precolombinas quedaron quebrantadas debido al desplazamiento lingüístico y la glotofágia, lo que obliga a vivir en una lengua imperial (el español). Desde la hermenéutica, este fenómeno sociolingüístico conduce a la pérdida irreparable de lo que pudo ser la interpretación y sensibilidad al mundo, gracias a la posibilidad de "haber nacido en una o más lenguas, y haberlas tenido inscritas en el cuerpo" (Mignolo, 2013, p.303).

En consecuencia, con esta compleja situación sociolingüística, los procesos didácticos sobre interculturalidad que se producen en estos contextos en programas de lenguas extranjeras, se convierten, según nuestro criterio, en un desafío al que la didáctica no puede dar respuesta unívocamente y debe recurrir, entonces, a un diálogo filosófico cuyas fuentes provengan de América Latina. La primera tarea consiste en definir críticamente qué se entiende en este continente por interculturalidad, tomando en cuenta los complejos estados históricos, políticos, lingüísticos y educativos que se han surgido desde 1492. Para el efecto, debemos comenzar por tomar distancias con diferentes formas de colonialidad del saber que se desprenden de documentos como el 
MCER y, en su lugar, establecer una definición de interculturalidad, de manera que en ella confluyan las propuestas que la filosofía latinoamericana ha promovido, y que en un momento dado pueden convertirse en la ruta epistemológica que le dé autodeterminación didáctica.

La tarea no resulta sencilla, sobre todo, si se toman en cuenta las críticas que la concepción de interculturalidad ha recibido desde un enfoque filosófico. Fornet-Betancourt (2004) plantea para este propósito que intentar reflexionar desde América Latina conservando los clásicos y las escuelas europeas representa una limitante epistemológica. En su lugar, debe conformarse "una práctica de la filosofía que toma el contexto histórico de los países latinoamericanos como su punto de partida y también como referencia primera para explicar su sentido y legitimidad" (Fornet-Betancourt, 2004, p.17).

Sin embargo, al revisarse los trabajos sobre interculturalidad por parte de la filosofía latinoamericana, Fornet-Betancourt encuentra que no hay una tradición teórica en este sentido y tampoco práctico-reflexiva; además, en sus avances, la filosofía latinoamericana ha dado la espalda a la interculturalidad, con lo cual: "Su desarrollo no responde al reclamo de justicia cultural articulado en las luchas sociales y en los testimonios intelectuales mencionados. O sea, no se hace cargo de la interpelación intercultural continuando todavía cerrada en gran medida a la posibilidad de refundarse desde la diversidad cultural latinoamericana" (2004, p.20).

Para el autor, son cuatro las razones que han dejado en evidencia las fallas de la filosofía latinoamericana en el tratamiento de la interculturalidad. En primer lugar, la filosofía latinoamericana sigue atada por vicios de colonialidad a la aprobación que de ella puede recibirse de Europa y de Estados Unidos. Mientras persista esta dependencia epistemológica, la filosofía latinoamericana no podrá dar cuenta de una idea de interculturalidad que recoja toda la diversidad cultural del continente.

En segundo lugar, en la filosofía latinoamericana todavía se resiente con gran fuerza las huellas de la modernidad eurocéntrica. Una forma de evidenciar esta dependencia se manifiesta en la necesidad de esta filosofía de concebir el texto escrito como la base de los análisis a pesar de la larga tradición oral que existe en este continente.

La tercera razón que establece el autor es la incoherente necesidad de esta filosofía de expresarse en dos lenguas únicamente, el español y el portugués, 
no obstante, la enorme diversidad lingüística del continente (quechua, aymará, guaraní, náhuatl o kuna), un testimonio contundente de una precaria apertura intercultural que sigue aferrada a un bilingüismo hispano-luso.

La última razón que revela deficiencia por parte de la filosofía latinoamericana en el tratamiento de la interculturalidad se refleja en el reduccionismo cultural del mestizo, con lo cual se están desconociendo otras formas de ser y estar en este continente; así mismo, se está demostrando una evidente influencia de colonialidad por parte de la filosofía al marginar a otros como los indígenas y los afroamericanos.

No obstante, estas críticas develan que no hay otro camino para intentar hallar una autodeterminación intercultural en la didáctica de las lenguas-culturas extranjeras. Sin embargo, Fornet-Betancourt encuentra que la contribución filosófica de este contexto a partir de 1992 es interesante; para tal efecto, se propondrá un exordio a la interculturalidad latinoamericana en lenguas extranjeras en la tercera parte de este trabajo a partir de algunos filósofos, buena parte de ellos inscritos en el Grupo Modernidad/Colonialidad.

\section{¿Qué es interculturalidad para América Latina en contextos mayoritariamente monolingües?}

Walsh considera que la interculturalidad tiene dos caras: de un lado, la que se relaciona con diferentes formas de globalización del poder, del capital y del mercado, que no es pertinente para el contexto latinoamericano; $y$, de otro lado, la que se concibe como "un proyecto político de descolonización, transformación y creación" (2009, p.2). Adicionalmente, la autora dice que la interculturalidad es y adquiere sentido cuando se percibe como un proyecto educativo que tiene un énfasis pedagógico-político-crítico, y que apunta al objetivo freiriano de reorganizar la sociedad, de manera que se tomen distancias con las estructuras que racializan, inferiorizan y deshumanizan al ser humano.

Walsh entiende que la interculturalidad como proyecto debe atender las inquietudes étnico-culturales, al promover una relación dialógica entre las culturas; para, de este modo, desenmascarar el racismo y la exclusión, con la intención de que las sociedades sean justas, equitativas, igualitarias y plurales. La autora concibe el término de perspectiva relacional como el contacto, el intercambio y las relaciones entre unas y otras culturas en condiciones de igualdad o desigualdad. Esta es una percepción propia que se establece de 
manera general en los autores europeos que teorizan sobre didáctica de la interculturalidad en la enseñanza-aprendizaje de lenguas extranjeras, lo cual oculta el lado en el que subyace la realidad social en la que se advierte con frecuencia el conflicto, el poder, la dominación y, por supuesto, la colonialidad epistémica y ontológica.

Otra forma de comprender la interculturalidad es de tipo funcional, con lo cual Walsh determina que mediante ella se establece el diálogo, la convivencia y la tolerancia. Esta interculturalidad se ajusta a los intereses de las políticas neoliberales, puesto que expresa un supuesto social ejemplar, pero no admite que esa misma política engendre estructuras de asimetría y desigualdad social, política y cultural. Finalmente, la autora considera que la interculturalidad debe asumirse desde una perspectiva crítica, lo que conduce a admitir que en la sociedad hay no sólo diversidad o diferencia, sino también un problema estructural-colonial-racial. Lo anterior, se expresa en la jerarquización y exclusión de los pueblos indígenas y afrodescendientes, poniendo en la cúspide de la estructura a los blancos y a los blanco-mestizos. Este tipo de interculturalidad se construye desde abajo, desde la gente y se opone al modelo funcional y relacional.

Según Walsh (2009), este tipo de interculturalidad está por construirse, por lo cual, al referirnos al término en este trabajo, se le debe comprender como proyecto, lo que quiere decir que es una reconceptualización, una idea con arraigo político, social, étnico, ético, epistémico, en donde confluyen saberes, conocimientos provenientes de los indígenas, de los afrodescendientes, de los blancos, de los mestizos y de los blanco-mestizos occidentalizados.

De acuerdo con las reflexiones de la autora, al hacer referencia a la interculturalidad en el campo educativo en América Latina, es preciso relacionarla con el bilingüismo que une el plurilingüismo y la multietnicidad de las lenguas indígenas. Este proyecto educativo se concretó en 1982 como resultado de la reunión regional de especialistas en educación bilingüe en México. No obstante, para los efectos de esta reflexión, el bilingüismo o el plurilingüismo que proviene de estas experiencias sociolingüísticas y glotopolíticas no es apto para la didáctica de las lenguas-culturas extranjeras, toda vez que las lenguas en contexto educativo son las lenguas hegemónicas e imperiales (Mignolo, 2013) y no las lenguas ancestrales de los pueblos indígenas.

En concreto, según nuestro criterio, el proyecto sobre interculturalidad crítica previamente referido, sí es pertinente para el esquema didáctico de las 
lenguas-culturas extranjeras, puesto que resemantiza la didáctica de la interculturalidad contextualizando asuntos históricos, políticos, étnicos, éticos, sociales y necesariamente culturales desde una perspectiva latinoamericana, de manera que se asume que la interculturalidad no es un proyecto europeo, sino un asunto glotopolítico de este continente del que nos ocuparemos a continuación.

\section{Interculturalidad crítica y glotopolítica en América Latina}

La glotopolítica debe comprenderse desde las políticas lingüísticas. De acuerdo con Chardenet (2016), las políticas lingüísticas necesariamente ponen en escena preocupaciones sobre la internacionalización de las lenguas, al tomar en cuenta de manera esencial a los interlocutores, seres multidimensionales, definidos a partir de conceptos sustanciales como el homo politicus, homo sapiens, homo habilis, homo economicus. En cada contexto y cultura, los usuarios de las lenguas, según las incidencias antropo-sociolingüisticas, construyen una cosmovisión específica, lo que quiere decir, según Chardenet, que pretender un esencialismo desde una sola visión del mundo logra en consecuencia interpretar la globalidad y la diversidad de las culturas de manera improcedente.

En ese mismo sentido, el autor acepta que hay dos conceptos sobre internacionalización de lenguas: universalizar el aprendizaje de las lenguas, fenómeno educativo al que debe referirse la didáctica de las lenguas extranjeras, y universalizar una lengua, proceso histórico-político que en diferentes momentos de la historia de la humanidad ha querido constituirse mediante campañas colonizadoras e invasoras como lo sucedido en América Latina a partir de 1492. Sin embargo, Chardenet admite que en tal caso se estaría hablando de una lengua hipotética, situación lingüística inexistente hasta el momento y difícilmente previsible.

Así como es imposible pensar que pueda existir la universalización de una lengua, es absurdo imaginar un mundo sin diversidad. Según lo plantea el autor, fitogenéticamente hablando, el empobrecimiento de la diversidad genética conduce a una pérdida general de diversidad biológica y, por consiguiente, a una gran vulnerabilidad. Esa misma problemática puede plantearse a nivel intercultural: pretender una sociedad monocultural y monolingüe, en lugar de ser una ventaja, tiende a convertirse en una debilidad sociocultural y antropo-sociolingüística.

Una de las grandes inquietudes que plantean los teóricos de la decolonialidad es la expansión del inglés, la regresión de otras lenguas que pudiéramos 
llamar minoritarias, y las consecuencias epistemológicas de esta expansión lingüística. Chardenet (2016) explica este fenómeno de la siguiente manera: el desarrollo económico, político, demográfico y científico de los Estados Unidos con su puente europeo, el Reino Unido, ofrece una base sociológica enorme de autores y lectores para revistas como Nature and Science, lo que se explica no por asuntos de tipo lingüístico-racionales, sino por factores de orden mercantil macroeconómico, político, estructural-científico, que constituyen este dominio inevitable.

A su vez, podría explicarse entonces lo que sucede con la comprensión e interpretación de la interculturalidad según los predicados de autores anglosajones. Esta expansión epistemológica obedece a razones de tipo comercial, unidas a factores de orden político, económico, estructural-científico, lo que quiere decir que las propuestas teóricas o práctico-reflexivas no pudieran ser más consistentes que las latinoamericanas a pesar de las deficiencias filosóficas previamente expuestas.

Este fenómeno glotopolítico afecta indudablemente la comprensión y la interpretación que pueda construirse desde América Latina sobre la interculturalidad en lenguas-culturas extranjeras o internacionales como las define Chardenet. Sin embargo, para tal situación hay una solución que puede equipararse a lo que sucede con la publicación de artículos científicos entre países: la coautoría entre autores latinoamericanos, europeos, orientales y africanos, de manera que logren elaborar procesos de investigación en interculturalidad, produciéndose lo que Mignolo (2013) define como pensamiento fronterizo: propuesta epistemológica que consiste en construir proyectos conjuntos pese al conflicto histórico-hermenéutico-epistémico que los antecede. Santos (2010) denomina a este tipo de proyectos pensamiento posabismal, es decir, una ruptura con la modernidad occidental que busca establecer una perspectiva epistemológica y de aprendizaje que surja de lo que el autor denomina el sur global, a través de un tejido de conocimientos heterogéneos que se traduce en una ecología de saberes o interconocimiento, comprendido en una transición paradigmática.

Si en lenguas-culturas extranjeras se requiere de una didáctica que dé cuenta de una interculturalidad crítica con énfasis en la problematización latinoamericana que se estructura a partir de reflexiones históricas, políticas, étnicas, éticas y educativas con implicaciones globales y locales, se requiere del establecimiento de políticas lingüísticas ciertamente críticas, pero igualmente 
conciliadoras o fronterizas, con el fin de elaborar proyectos de investigación intercultural de amplia difusión epistémica.

\section{Desafíos filosóficos de la interculturalidad en América Latina}

Hasta el momento, hemos establecido tres presupuestos básicos, a propósito de una autodeterminación intercultural en la didáctica de las lenguas-culturas extranjeras para América Latina. Por un lado, hemos dicho que el continente, didácticamente hablando, debe desligarse de la epistemología eurocéntrica que define la interculturalidad como una expresión de colonialidad del saber de tipo funcional que no es pertinente para este contexto mayoritariamente monolingüe. Por otro lado, hemos reconocido que pese a las debilidades de la filosofía latinoamericana para dar respuesta a los vacíos teóricos o práctico-reflexivos interculturales, hay que construir propuestas afirmadas en esta filosofía si se desean establecer límites decoloniales con Europa y el mundo anglosajón. Finalmente, hemos establecido que sí es posible autoderminarse didácticamente en asuntos crítico-interculturales que han afectado a los pueblos latinoamericanos, especialmente a los indígenas y a los afrodescendientes que, para tal efecto, precisa establecer principios glotopolíticos que den solidez y estructura fronteriza a las investigaciones que se deseen hacer desde América Latina en proyectos posabismales que permitan la expansión global de dichas propuestas.

Consecuentes con esos presupuestos, es importante ahora dimensionar y reflexionar sobre los desafíos filosóficos que debe afrontar la interculturalidad y su construcción didáctica desde el contexto latinoamericano.

\section{El latinoamericanismo}

El primero de esos desafíos es confrontar lo que Mendieta (2006) denomina latinoamericanismo, una resemantización del orientalismo de Edward Said. Para Mendieta, el latinoamericanismo tiene cuatro caras que se resumen en las formas como América Latina ha sido definida de acuerdo con los criterios de sí misma, de los Estados Unidos, de Europa y de los latinos que viven en Estados Unidos. El primer tipo de latinoamericanismo de Mendieta es el que se define como la lucha cultural en la que se manifestó el imperialismo estadounidense sobre este territorio. El segundo tipo es el que se denomina latinoamericanismo de estudios de área, que se caracteriza por el afán de los países del primer mundo en recoger y difundir conocimiento sobre los 
países del tercer mundo, de donde surge la supervisión epistémica que hoy se traduce por los grupos decoloniales como colonialidad del saber. El tercer lationamericanismo es el llamado latinoamericanismo crítico que pone a Latinoamérica en oposición a los Estados Unidos con énfasis antiimperialista, anticapitalista; Mendieta lo define como un marxismo con cara de indígena. El cuarto y último tipo de latinoamericanismo es el que se construye por los latinoamericanos que desde tiempo atrás se instalaron en Estados Unidos. Desde allí buscan criticar la homogenización de América caracterizada por una globalización en la que Latinoamérica queda presa de la dialéctica del hemisferio norteamericano.

Este desafío filosófico, de evidente complejidad para la estructuración de una interculturalidad en lenguas-culturas extranjeras, consiste en diseñar un proyecto de interculturalidad crítica (Walsh, 2009) que logre encontrar una identidad latinoamericana coherente con sus pretensiones; dicho de otra manera, que no caiga en un marxismo indigenista a ultranza, instrumentalizando a los pueblos indígenas y afroamericanos en procura de un proyecto que resulte ser tan excluyente y xenófobo como el que se advierte en los comportamientos de ciertos grupos o partidos políticos norteamericanos y europeos.

\section{Mimetismo e hibridación}

Es Bhabha quien establece estos conceptos para referirse al tema colonial. Según lo plantea Vega (2010), el mimetismo bhabhiano se caracteriza por tener la cualidad de reproducir los planteamientos estructurales del colonizador al interior de los colonizados, engendrando un grupo de colonizados que imiten al colonizador, con lo cual se facilita la tarea de ejercicio del poder sobre los subalternizados. La escuela, según lo plantea Fanon, es la estrategia más efectiva para lograr crear deseos de blanquearse en quienes hacen las veces de colonizados. Lo dicho hasta aquí supone que para los efectos de la autodeterminación intercultural en lenguas-culturas extranjeras es un desafío crear distancias conceptuales respecto de los modelos teórico-eurocéntricos previamente establecidos con Walsh (2009). En concreto, dichas distancias conceptuales se perciben como todo un reto considerando que evitan re-crear un proyecto de didáctica intercultural con el objetivo de que estos remeden o reproduzcan, como se menciona al inicio del texto, lo que fue ya preestablecido por los autores que se expresan sobre didáctica intercultural en textos como el MCER o el CARAP, cuyas estrategias son funcionales o relacionales, permitiendo divisar un estrecho marco donde dicho eurocentrismo 
limita la didáctica intercultural en ejercicios ciertamente académicos cuando a dicho propósito se refiere.

La mimesis no concluye allí, se comprende además como la estrategia del colonizado para desobedecer al colonizador, de manera que en el discurso del jerarquizado aparece lo que le fue negado; es decir, la negación colonial, pero con características de hibridación, una resemantización o reinterpretación conceptual. Este desafío de hibridación bhabhiana debe revisarse cuidadosamente a la hora de producir la autodeterminación intercultural en la didáctica de las lenguas-culturas extranjeras. Al elaborarse el proyecto de interculturalidad crítica, las nociones preestablecidas por las propuestas eurocéntricas deben ser analizadas, de manera que pueda re-crearse una nueva idea intercultural que interrogue desde dichas nociones el concepto de cultura e interculturalidad, elemento epistemológico que conduciría a una hibridación intercultural para América Latina.

\section{Una filosofía intercultural en América Latina}

Implementar críticamente una corriente filosófica que desde Latinoamérica hable de una filosofía intercultural es una acción reflexiva. Fornet-Betancourt afirma que la filosofía de este continente tiene deudas pendientes con la interculturalidad, de manera que introducir la idea de filosofía intercultural en América Latina se convierte en un desafío tan complejo como cuestionable. Sin embargo, si la interculturalidad en didáctica de lenguas-culturas extranjeras, como diría Chardenet, desea desprenderse de la subalternización en que se ha mantenido con respecto a Europa, se hace necesario entonces revitalizar una filosofía intercultural.

Salas define la filosofía de la interculturalidad como

una alternativa para transformar nuestros modos de practicar la filosofía, es decir, para 'provocar' un cambio de rumbo de la filosofía, al defender, precisamente, la recontextualización del quehacer filosófico en los mundos de vida y en las culturas vivas de 'la gente', y al propugnar, además, como consecuencia de lo primero, que todo ejercicio sabio de la inteligencia humana [latinoamericana] no puede culminar en la razón (...) sino que tiene que (...) posicionarnos éticamente. (2006, p.8)

Salas incluye la ética como elemento base de su definición. Con ello pone de manifiesto que se debe promover una transformación de la filosofía que tienda al reencuentro y al diálogo de las culturas para así comprender la diversidad 
de la humanidad. Al tomar como fundamento la diversidad humana, el autor liga la filosofía intercultural con el presente, el pasado, las tradiciones, las culturas y compromete lo que denomina un diálogo entre culturas y entre etnicidades que apunte a una convivencia solidaria.

$\mathrm{Al}$ hacer referencia a las etnicidades como marco fundamental para intentar una filosofía intercultural, se genera consecuentemente una ética intercultural. Esta propuesta es no sólo pertinente, sino difícil de gestionar a nivel filosófico en un mundo que, como lo reconoce el autor, se encuentra en una grave crisis moral. En ese mundo, y concretamente en el latinoamericano, la crisis moral se expresa en los comportamientos de la gente, en los resultados de la gestión política de sus gobernantes profundamente corruptos y en los excesos de poder de los países ricos que se empeñan en continuar aprovechándose de los recursos naturales y humanos de estos territorios. Por consiguiente, como lo reconoce Salas, la ética intercultural le pertenece a América Latina, que interroga y cuestiona el modelo económico hegemónico que impera en el mundo y que deja serias heridas en estos pueblos.

En efecto, una filosofía intercultural está en la obligación de dar cuenta de un ethos latinoamericano capaz de explicar la actividad humana entendida como la respuesta a las necesidades de los seres que la habitan. Es justamente en la acción humana donde radica el mayor desafío para la filosofía latinoamericana en la construcción de una interculturalidad crítica para la didáctica en lenguas-culturas extranjeras: los fenómenos relativos a la identidad, a través de los cuales la filosofía intercultural se convierte en la propuesta esencial de la ética, pues constituye la realización auténtica de los latinoamericanos. Al promoverse una filosofía intercultural cuyo eje central se encuentra en la ética intercultural, la didáctica de las lenguas-culturas extranjeras para América Latina debe dar cuenta de los elementos que definen los referentes identitarios, instaurando criterios relacionados con la axiología, la epistemología, la ontología y la antropología cultural.

\section{Exordio para una interculturalidad crítica en lenguas-culturas extranjeras en América Latina}

En consecuencia de los planteamientos previos, presentamos un preámbulo de la interculturalidad crítica para las lenguas-culturas extranjeras en América Latina. La propuesta está estructurada en cuatro momentos epistemológicos para contextos mayoritariamente monolingües como el latinoamericano, sus pretensiones 
son la fase introductoria para la construcción de un proyecto común de diferentes grupos o entes investigadores interesados en este proyecto intercultural.

\section{EI encubrimiento del otro}

Históricamente, la interculturalidad para América Latina debe resignificarse a partir de la Modernidad como proyecto que, según Dussel (1994), nace cuando Europa se da cuenta de que puede confrontarse con un "otro" en

quien puede ejercer control y violencia. Ese primer momento histórico de reflexión intercultural para América Latina surge cuando el hombre europeo desarrolla lo que Dussel denomina el ego-conquiro, parodiando el ego-cogitans cartesiano. Ese ego conquiro que se implementa con la Modernidad se produce a partir de 1492, momento en que según el autor se produce el en-cubrimiento de una alteridad, concretamente la de lo no-europeo.

Resultado de ese momento histórico, Dussel (1994) reclama una relación histórico-hermenéutica que permita una comunicación intercultural a través de la cual se redescubra al oprimido, al incomunicado, al excluido que por siglos ha estado postrado a la condición subalterna con respecto a una epistemología europea que hoy todavía se apropia de un discurso que pretende definir globalmente lo que se entiende por interculturalidad, desconociendo un pasado que aún se resiente en el presente.

En la actualidad, no se ven todavía los aportes latinoamericanos a la interculturalidad en lenguas-culturas extranjeras. Podría considerarse que las contribuciones que pudieran venir del resto del mundo, de América Latina, por ejemplo, en la discusión de interculturalidad no merecieran espacio alguno. Mediante la construcción y posterior difusión mundial del MCER y de la forma como se consolida la interculturalidad o la competencia intercultural, subsiste la intención de encubrir al "otro", persistiendo en la idea de negar un pensamiento fronterizo (Mignolo, 2013) intercultural, en el cual coincidan zonas de contacto para la diversidad epistemológica, principio fundamental de dicho concepto.

\section{Geopolítica y legados coloniales del conocimiento}

No hay intención de resignificación geohistórica, geográfica, geocultural o geocognitiva en los postulados didácticos y epistemológicos de interculturalidad provenientes de Europa para el proceso de enseñanza-aprendizaje de las lenguas extranjeras. Geoculturalmente, el modelo a seguir es el MCER, 
en cuya racionalidad intercultural caben únicamente los países europeos. En esta, no hay forma de ejemplificar la superación de los estereotipos que socavan la identidad de los pueblos latinoamericanos, como suele ocurrir con la imagen deteriorada de los colombianos, a quienes se les considera irremediablemente narcotraficantes.

Así, por ejemplo, Kant no tuvo ningún reparo en afirmar que la única raza capaz de progresar es la blanca europea. Esta forma de subalternizar al indio, al negro y al mestizo en una lógica perversa que histórica y geográficamente lo ubica en condición inferior, debe reordenarse y resignificarse. Para lograrlo, tal como lo sugiere Walsh (2005) la interculturalidad debe abordarse desde un principio ideológico que se perfila en una política lingüística epistémica (glotopolítica epistémica) cuyas consecuencias se advierten en una interculturalidad de la cual se concluya que desde el Sur también se construyen conocimientos. Como revela Dussel (2005), una experiencia clara es la ecuatoriana al establecer un modelo educativo de formación científica intercultural a través de la cual se superan las asimetrías que, tradicionalmente, y desde el "encubrimiento" de América se ha diseñado por cuenta de la colonialidad del saber.

\section{Hybris de punto cero}

Suponer que solamente la interculturalidad es epistemológica y didácticamente sólida, que legitima el conocimiento mientras provenga de Europa o de los países anglosajones, es el resultado de una tradición epistémica que se ha instalado desde que la Modernidad como proyecto se consolidó a nivel universal. Por consiguiente, otras formas posibles de recrear, interpretar y pensar el mundo deben quedar en el punto cero, es decir, no existen. En otras palabras, Europa se apropia del derecho a denominarse centro étnico y cultural (Castro-Gómez, 2005). La hybris del punto cero de Castro-Gómez se comprende al revisar las campañas conquistadoras de España en cuyo propósito se estableció una taxonomía de Lebenswelt, o mundo de la vida, que negaba otras formas de estar en el mundo mientras no fueran similares a las españolas.

En este ejercicio intercultural, la hybris del punto cero en la didáctica de las lenguas extranjeras se instituye en la comprensión de interculturalidad $\mathrm{y}$, muy probablemente, ni siquiera son los europeos autores de las concepciones de interculturalidad los responsables de esa hybris, son los mismos 
latinoamericanos quienes bajo el peso de la colonialidad del saber se consideran no aptos para generar ideas propias que disten de lo que para Europa es lo epistemológicamente válido. En consecuencia, se debe diseñar una ideología para América Latina en la cual los referentes identitarios de los estudiantes en contexto mayoritariamente monolingüe sean el objetivo fundamental en la didáctica de las lenguas-culturas extranjeras, tal y como se construye en Ecuador la interculturalidad como ideología y proyecto educativo de formación científica.

\section{Interculturalidad crítica en contexto mayoritariamente monolingüe}

Probablemente, el error epistemológico más grande que pueda atribuirse a la didáctica en las lenguas-culturas extranjeras sea la postura acrítica que se asume en ese contexto en relación con lo que se sugiere desde Europa para la concepción y comprensión de la interculturalidad entendida como competencia. Esto quiere decir que la competencia comunicativa intercultural debe revisarse según el contexto lingüístico, histórico, político, geopolítico, geocultural, glotopolítico y educativo que afecta al contexto mayoritariamente monolingüe latinoamericano. La diversidad cultural es tan amplia como lo es el mundo, y pretender establecer criterios de hybris de punto cero para administrar una didáctica universal de la interculturalidad no es solamente un proyecto osado e irrespetuoso, sino que además es una expresión clara de colonialidad del saber didáctico en lenguas extranjeras.

En el ejercicio de negación de una hybris intercultural eurocéntrica, debe partirse de una inflexión intercultural en cuyo propósito se reconozca la diversidad cultural del mundo entero y, especialmente, para los propósitos de esta discusión de América Latina. En ese propósito, Fornet-Betancourt establece que la interculturalidad debe comprenderse no como un diálogo entre culturas ni como una posición teórica que encaja fácilmente en lo que desde Europa se prescribe, sino como "una postura, una disposición por la que el ser humano se capacita (...) y se habitúa a vivir 'sus' referencias identitarias en relación con los llamados 'otros', es decir, compartiéndolas en convivencia con ellos" (2004, p.14).

Fornet-Betancourt considera la interculturalidad como un proceso de reaprendizaje y de reubicación en la que el ser humano busca un contexto. Para el autor, interculturalidad es en conclusión "el reconocimiento de la necesidad de que una dimensión fundamental de la práctica de la cultura que 
tenemos como 'propia' debe ser la de la traducción de los 'nombres propios' que consolidan su tradición" (2004, p.15). Esto quiere decir que, al intentar una semántica propia para la interculturalidad latinoamericana, se hace indispensable el contacto de formas de entender, vivir el mundo e interpretarlo, lo que necesariamente conduce a la descentración del lebenswelt en el que se ha venido viviendo para reinterpretarlo y apropiárselo a partir de una construcción epistémica fronteriza y que aquí llamaremos interculturalidad fronteriza crítica.

\section{Conclusiones}

La didáctica de las lenguas extranjeras en contextos mayoritariamente monolingües es ciertamente compleja, debido a la omnipresencia de la lengua materna en estudiantes que desean aprender otras lenguas. El espacio-tiempo para que la lengua extranjera se convierta en esos contextos en acción social es casi imposible: el estudiante o actor social no se constituye en usuario de la lengua sino en los espacios estrictamente académicos, los contextos sociales de los cuales dispone no son auténticos, de manera que los docentes deben re-inventarlos para bien de las competencias lingüísticas y pragmáticas que debe desarrollar el aprendiente.

A contrario sensu, la interculturalidad está al orden del día, no obstante el contexto mayoritariamente monolingüe. La misma cultura, y los propios referentes identitarios de los cuales dispone el estudiante y sus pares, se constituyen en agentes socialmente dinámicos para que se lleve a cabo una praxis intercultural en la cual el estudiante de lenguas extranjeras sea capaz de cuestionar su propio entorno y el de los demás. La ausencia de una otredad europea o anglosajona no es impedimento para que interculturalmente no desarrolle lo que el MCER denomina consciencia intercultural. Ciertamente, lo ideal sería que contara con un "otro" diametralmente opuesto no sólo cultural, sino étnica e identitariamente diferente.

Si bien la problemática en cuestión es la ausencia de espacio y tiempo de diálogos y contactos interculturales, el verdadero problema radica en las evidencias latentes de colonialidad epistemológica o del saber que se han universalizado para didactizar la interculturalidad que terminan por generar colonialidad ontológica. Mientras persista en América Latina una comprensión de lo que es competencia intercultural desde parámetros epistémicos de Europa, los postulados de América Latina para la didáctica intercultural 
no serán los que convengan y legitimen. En esas condiciones el estudiante latinoamericano entrará en contacto con el otro europeo a partir de unos predicados eurocéntricos que suponen que no podemos crear una idea propia de interculturalidad y de su respectiva didáctica, lo que Santos (2010) denomina el pensamiento abismal.

El estudiante latinoamericano de lenguas extranjeras debe constituir en su competencia intercultural la capacidad para mediar con la diferencia, hacerse un gestor intercultural y ser consciente de una ideología intercultural fundada en "el susodicho" descubrimiento de América que le viene dado como hecho natural. Adicionalmente, debe comprender que geopolítica, geocultural, glotopolítica y sociolingüísticamente es heredero de una historia que ha venido negándole su capacidad de generar un conocimiento propio, que le permita interpretar el mundo desde una perspectiva científica con la cual construir una idea de interculturalidad epistémica.

Por otro lado, esa competencia intercultural latinoamericana en lenguas extranjeras debe darle la certeza al estudiante de esas lenguas, pues no hay ninguna razón para considerar que la forma de vivir el mundo y de estar en el mundo no debe subsumirse a la europea, porque tradicionalmente viene aplicando desde 1492 el principio de hybris mediante el cual se niega la certeza de mundo de la vida y de referente identitario tan válido como el europeo.

Finalmente, el estudiante de lenguas extranjeras, al desarrollar la competencia intercultural, debe pasar por un proceso de inculturación como un elemento sustancial para la toma de consciencia del desafío intercultural. Sólo de ese modo puede concebirse la idea de un profesional de lenguas modernas capaz de reinterpretar su propia historia y de reubicarla en un mundo que no le puede seguir negando la idea de ser epistemológica y ontológicamente viable para el futuro.

\section{Referencias}

Calvet, L-J. (1999). En Montoya, A. (2013). La incidencia de las políticas y la planeación en las actitudes lingüísticas de los estudiantes colombianos. Forma y función 26(1), pp. 237-260. 
Chardenet, P. (2016). Internationalisation de l'enseignement supérieur et de la recherche. Pourquoi une politique linguistique stratégique en contexte latino-américain? Revue de la SAPFESU, 39, pp. 14-45.

Candelier, M. (Coord.). (2012). Le CARAP. Austria: Consejo de Europa.

Castro-Gómez, S. (2005). La hybris del punto cero. Bogotá: Editorial Pontificia Universidad Javeriana.

Consejo de Europa (2002). Marco común europeo de referencia para las lenguas: aprendizaje, enseñanza, evaluación. Madrid: Artes gráficas.

Dussel, E. (1994). 1492: el encubrimiento del otro, hacia el origen del mito de la modernidad. La Paz: Plural Editores.

Fornet-Betancourt, R. (2004). Crítica intercultural de la filosofía latinoamericana actual. Madrid: Editorial Trotta.

Lázár, et al. (2007). Évaluer la compétence communicative interculturelle. Strasbourg: Conseil de l'Europe.

Martínez, F. J. (2009). Ontología y diferencia: la filosofía de Gilles Deleuze. Eikasia, IV (23), pp. 33-335.

Mendieta, E. (2006). Ni orientalismo ni occidentalismo: Edward Said y el latinoamericanismo. Tabula Rasa, 5, pp. 67-85.

Mignolo, W. (2009). El lado más oscuro del renacimiento. Universitas humanística, 67, pp. 165-203.

Mignolo, W. (2013). Historias locales / diseños globales. Madrid: Ediciones Akal, S.A.

Montoya, A. (2013). La incidencia de las políticas y la planeación en las actitudes lingüísticas de los estudiantes colombianos. Forma y función, 26(1), pp. 237-260.

Plaza, P. (2016). Lenguas en peligro de extinción y acciones para su revitalización. PROEIB Andes. Revisado el 15 de abril de 2018, en http:// www.proeibandes.org/ciei/lineas $\% 20 \mathrm{de} \% 20$ investigacion/Pedro\%20 Plaza.pdf 
Salas, R. (2006). Ética intercultural. Ensayos de una ética discursiva para contextos culturales conflictivos. (Re) Lecturas del pensamiento latinoamericano. Quito: Ecuador, Ediciones Abya-Yala

Santos, B. (2010). Descolonizar el saber, reinventar el poder. Montevideo: Ediciones Trilce.

Vega, M.J. (2010). Homi Bhabha. Webislam. Revisado el 15 de abril de 2018, en https://www.webislam.com/articulos/38131-homi bhabha.html

Walsh, C. (2005). Interculturalidad, conocimientos, decolonialidad. Signo y Pensamiento, 24(46), pp. 39-50.

Walsh, C. (2010). Interculturalidad crítica y educación intercultural. Construyendo Interculturalidad Crítica, 75-96. Revisado el 15 de abril de 2018, en https://scholar.google.com.co/scholar?q=Walsh+interculturalidad $+\mathrm{cr} \% \mathrm{C} 3 \%$ ADtica $+\mathrm{y}+$ educacion + intercultural\&hl $=$ es \&as $\mathrm{sdt}=0 \&$ as_vis $=1 \&$ oi $=$ scholart\&sa $=$ X\&ved $=0$ ahUKEwi5m7_esczYAhWkk-AKHTZ4DQsQgQMIJDAA 